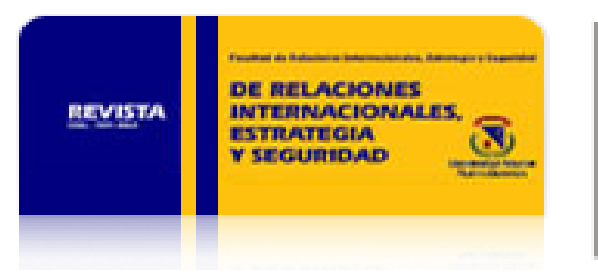

Revista de Relaciones Internacionales, Estrategia y Seguridad

ISSN: 1909-3063

cinuv.relinternal@unimilitar.edu.co

Universidad Militar Nueva Granada

Colombia

Sánchez Cabarcas, Fabio

WALTZ Y KEOHANE SOBRE EL 11-S Y LA INTERVENCIÓN EN IRAK

Revista de Relaciones Internacionales, Estrategia y Seguridad, vol. 4, núm. 2, julio-diciembre, 2009,

pp. 67-76

Universidad Militar Nueva Granada

Bogotá, Colombia

Disponible en: http://www.redalyc.org/articulo.oa?id=92712972006

- Cómo citar el artículo

- Número completo

- Más información del artículo

- Página de la revista en redalyc.org

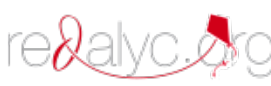

Sistema de Información Científica

Red de Revistas Científicas de América Latina, el Caribe, España y Portugal

Proyecto académico sin fines de lucro, desarrollado bajo la iniciativa de acceso abierto 
rev.relac.int.estrateg.segur.4(2):67-76,2009

\title{
WALTZ Y KEOHANE SOBRE EL 11-S Y LA INTERVENCIÓN EN IRAK
}

\author{
Fabio Sánchez Cabarcas*
}

\section{RESUMEN}

Este artículo revisa las posturas de Kenneth Waltz y Robert Keohane sobre las consecuencias de los atentados terroristas del 11-s en Nueva York y la intervención militar estadounidense en Irak desde 2003. En la primera parte se estudia el análisis neorrealista de Waltz, y la fuerte crítica que hace a la respuesta de la administración Bush. Posteriormente, se encuentra el análisis neoinstitucionalista que hace Keohane de los fenómenos estudiados, criticando la falta a la normatividad e institucionalismo internacionales por parte de Washington. Finalmente, algunas conclusiones sobre estas interpretaciones.

Palabras clave: Kenneth Waltz, Robert Keohane, neorrealismo, neoinstitucionalismo, teoría de relaciones internacionales, 11-s, Guerra de Irak 2003.

* Comunicador Social-Periodista, PUJ, Magíster en Relaciones Internacionales, PUJ, Doctorando en Relaciones Internacionales, Universidad Autónoma de Barcelona. Profesor de Tiempo Completo, Director (E) Departamento de Relaciones Internacionales, Universidad Sergio Arboleda - Bogotá. fabiob.sanchez@usa.edu.co 


\begin{abstract}
This article reviews the postures assumed by Kenneth Waltz and Robert Keohane regarding the consequences of the terrorist attacks of 9-11 on New York and the ensuing U.S. military intervention in Iraq. The first part considers the neo-realist analysis of Kenneth Waltz and its pointed criticism of the Bush administration's response to the attacks. Subsequently, the article examines Robert Keohane's neo-institutional analysis of the attacks, in which he criticizes the lack of a normative and institutionalist framework evidenced by Washington policy-making. Lastly, some conclusions are offered about these interpretations.
\end{abstract}

Key words: Kenneth Waltz, Robert Keohane, neo-realism, neo-institutionalism, international relations theory, 9-11, 2003 Iraq War.

\title{
INTRODUCCIÓN
}

La teoría es importante como ayuda y guía en la búsqueda de respuestas a los fenómenos sociales. En el campo de las relaciones internacionales, la teoría aspira a hacer más comprensibles algunos sucesos del ordenamiento mundial; en ocasiones, se trata de ir más allá de la simple descripción para llegar a explicaciones causales o predicciones de los fenómenos observados, teniendo en cuenta algunos acontecimientos del pasado (Viotti y Kauppi, 1987: 3). Incluso, Keohane hace 20 años, manifestaba en su obra ya clásica Neorealism and it 's Critics: "Lo que necesitamos ahora es entender el cambio pacífico mediante la combinación de análisis académicos multidimensionales, con formas más visionarias de ver el futuro» (Keohane, 1986: 200).

En este trabajo se pretende revisar el análisis que hacen Kenneth Waltz (neorrealismo) y Robert $O$. Keohane (neoinstitucionalismo), sobre las consecuencias del 11-s y la posterior intervención en Irak para el sistema internacional. Se han seleccionado estos dos autores debido a su trascendencia y aporte a la teoría de las RRII (Relaciones Internacionales), trabajo que sería muy extenso de citar, y no es propósito de este ejercicio. ${ }^{1}$ El debate entre estas dos escuelas se ha venido desarrollando desde hace aproximadamente 15 ó 20 años. Estas teorías representan paradigmas que pueden moldear las imágenes del mundo, y asimismo, influenciar las prioridades en la investigación y también en las decisiones y debates políticos. (Lamy, 2001: 184). Ambas corrientes son racionalistas y han conformado el «núcleo duro» de la disciplina, es decir, el mainstream de las RRII en los últimos 20 años (Sodupe, 2003: 148), sobre todo en cuanto a la definición de la agenda de estudio que tiene que ver con los procesos de globalización a través de la gobernanza mundial y el análisis de temas de seguridad luego del 11-s. (Barbé, 2007: 81). Asimismo, se debe tener en cuenta la afirmación de Weaver de que a pesar de que la síntesis neo-neo puede resultar algo exagerada, ha tenido la capacidad de establecer un programa de investigación racionalista (Weaver, 1996), ya que todos comparten un compromiso con una orientación positivista de la ciencia (Sodupe, 2003: 148).

${ }^{1}$ Para una revisión parcial de la vida y obra de esto autores, véase (Griffiths et al., 1999).

WALTZY KEOHANE SOBRE EL 11-SY LA INTERVENCIÓN EN IRAK 
El trabajo se desarrolla de la siguiente manera. En primer lugar, se encuentra el análisis de Waltz, precursor del realismo estructural y de los sistemas de equilibrio de poder (Waltz, 1979, 1988, 2000). Posteriormente, se analiza la postura de Keohane máximo exponente del neoinstitucionalismo (Keohane y Nye, 1977, Keohane, 1982, 1988,1998), quien explica cómo luego del 11-s, se pisoteó a las instituciones internacionales y se falló en contrarrestar a lo que él denomina «la globalización de la violencia informal». Finalmente, se encuentran las conclusiones.

\section{WALTZ SOBRE EL 11 - S E IRAK}

Para Waltz las decisiones de la Administración Bush, luego de los ataques del 11-s, deben revisarse con especial atención. Según este autor, el uso que se ha dado al concepto (preemption) -anticipación- es confuso. En términos de estrategia militar serviría, siempre y cuando se tenga certeza de que el enemigo atacará en el futuro. Pero en el caso de Irak, la información disponible demostraba que ese país era débil, que Hussein no atacaría a nadie, ni siquiera a sus vecinos (Waltz, 2003). Los trágicos efectos de las sanciones impuestas por la ONU, después de 1991, constatan este análisis, por ejemplo, el PIB de Irak era de US\$ 15 billones, mientras que el gasto solamente militar de EE.UU. alcanzaba los US $\$ 400$ billones, lo cual lleva al autor a manifestar: «Irak es muy débil, más de lo que lo era en 1991, cuando peleamos la Guerra del Golfo, y lo sabíamos» (Waltz, 2003). De la misma manera, si se hablara de -prevención- también sería equivocado, pues este concepto opera sobre el tiempo, y se trata de atacar al adversario antes de que sea más fuerte y la guerra sea más difícil, y como vimos, Irak nunca podría ser más fuerte que EE.UU. No obstante, el conflicto se tornó más crítico debido a que la guerra de guerrillas irregular y urbana no es fácil para ejércitos que, como el estadounidense, están preparados para el combate convencional.

En el mismo orden, Waltz critica que la administración Bush manifestaba que la contención y la disuasión no funcionaban contra Saddam Hussein; el propio Presidente Bush decía: «No confío en la cordura de Hussein», cuando la historia demuestra que había sido contenido, y es el mismo teórico quien arguye: «¿Cómo puede alguien sobrevivir en un mundo difícil? Es mucho más difícil que ganar una reelección en EEUU.» (Waltz, 2003).

Por otra parte, Waltz cree que Hussein siempre quiso tener armas nucleares. En política exterior siempre cuentan los antecedentes; la destrucción de la planta de Osirak, en 1981, por parte de los israelíes, es una prueba de ello. ${ }^{2}$ No obstante, cuando logró tener algunas armas químicas, no las compartió, las usó contra los kurdos, shiítas e iraníes; además, tenía una capacidad reducida que se agotó casi por completo con las sanciones de la década de 1990. Lo anterior desvanece el argumento que los neocons esgrimieron ante el Consejo de Seguridad de la ONU sobre la supuesta relación entre Al Qaeda y Hussein.

${ }^{2}$ Para más información al respecto véase: (Raas y Long, 2007). 
Por otra parte, el autor considera que la disuasión tradicional no funciona si grupos terroristas adquieren WMD (Weapons of Mass Destruction). Explica que el problema está en la percepción clásica de tener un enemigo "físico" y "con dirección» sobre el cual se puede tomar retaliación, tal y como ocurrió con Irak, en cambio los terroristas no se pueden encontrar, no se sabe en dónde están (Waltz, 2003). Esta dificultad fue uno de los principales desafíos de la administración Bush, puesto que siempre ha sido complejo tratar de impedir que estados fallidos y grupos terroristas compren WMD, más ahora que estos han identificado que la única forma de disuadir al hegemón es mediante esta herramienta, y además ya tienen claro que no pueden competir en escenarios de conflicto tradicionales, ni siquiera Rusia o China (Waltz, 2003).

En palabras del propio Waltz:

"Cualquier tonto puede ver que la única forma de disuadir a los Estados Unidos es con armas de destrucción masiva. No puedes competir con armamento convencional. Eso es absolutamente imposible. Rusia no lo puede hacer. China no lo puede hacer. Obviamente, para estos rogue states, es sólo una fantasía. Ni siquiera podrían intentarlo, ¿Cierto? Así que si creen que su seguridad está directamente amenazada, en particular por los EE.UU., o los EE. UU. actuando conjuntamente con otros países del área, van a hacer todo lo que puedan para adquirir armamento disuasivo nuevamente, la mejor opción será el armamento nuclear-» (Waltz, 2003).

En el mismo orden, Waltz identifica tres aspectos básicos en la política internacional desde el 11-s: I) el gran desequilibrio de poder (luego del ocaso de la URSS), II) la proliferación gradual de WMD y, III) la permanencia y prevalencia de crisis mundiales en las que está envuelto EE.UU. (Waltz, 2002), citado en (Kennedy-Pipe y Renger, 2006: 542). Lo anterior constituye un análisis que guarda coherencia con los postulados clásicos de Waltz, quien junto con Scott Sagan, a principios de la década de 1980, manifestó que las armas nucleares se dispersarían por el mundo, debido a que las unidades del sistema, a pesar de tener los mismos atributos, buscaban tener las mismas capacidades, más allá del estado de anarquía y la estructura organizadora del sistema (Waltz et al., 1995).

\section{KEOHANE 11- S Y LA GLOBALIZACIÓN DE LA VIOLENCIA INFORMAL}

Antes de pasar al análisis de los propios autores, se debe tener en cuenta que el estudio de regímenes e instituciones ${ }^{3}$ es fundamental en el campo neoliberal, razón por la cual los siguientes autores consideran a la intervención estadounidense en Irak como una fuerte violación a las normas y

\footnotetext{
${ }^{3}$ Se puede afirmar que las definiciones sobre instituciones y regímenes de la escuela neo-institucionalista son muy similares, veamos unas de las más representativas. Instituciones: «Son vistas como reglas y prácticas conectadas, que prescriben roles, limitan actividades y moldean las expectativas de los actores. Las instituciones pueden incluir organizaciones, agencias burocráticas, tratados y acuerdos y prácticas informales que los estados aceptan cumplir. Por ejemplo, el equilibrio de poder que los estados aceptan cumplir en el sistema internacional es una institución». Adaptado de Haas, Keohane y Levy en: Institutions for the Eartht (Haas et al., 1993, citado en, Lamy, 2001: 189).
}

WALTZY KEOHANE SOBRE EL 11-S Y LA INTERVENCIÓN EN IRAK 
regularidad del sistema internacional, un campo de debate que algunos, como Keohane, consideraban ganado desde la academia:

«Es muy decepcionante desde un punto de vista profesional, porque cuando nosotros, que enfatizamos y valoramos el papel de las instituciones en la política global creemos que hemos ganado el debate (que creo que lo ganamos intelectualmente, en la academia), es decepcionante presenciar estos retrocesos -personas (algunas viejas, como Rumsfeld; otras no tan viejas, como Rice) que simplemente no lo captan. Un académico, formando a personas durante veinte años esperaría, por lo menos, que aprendieran algunas de estas nuevas realidades. -Repito- es decepcionante» (Keohane, 2004).

Para este autor, los hechos del 11-s generaron consecuencias incalculables para la política doméstica y mundial; en la primavera de 2003 manifestó la imposibilidad de hacer predicciones, y de la misma manera sostuvo que estos hechos constituían un amplio desafío para los modelos analíticos tradicionales que deben dar respuesta a estos sucesos y que, según el autor, son insuficientes porque ignoran el impacto de la religión en la política mundial, a pesar de que ésta es un elemento presente en el pensamiento realista clásico (Keohane, 2002: 29).

Así pues, Keohane propone el concepto de "la globalización de la violencia informal» y no del terrorismo ante la complejidad que implica definir este fenómeno. ${ }^{4}$ De la misma manera, propone

Por su parte, la definición de régimen más utilizada es la de Krasner: «Normas, reglas, principios y procesos de toma de decisiones, al rededor de los cuales convergen las expectativas de los actores en un área determinada de RRII» (Krasner, 1983). Asimismo, Young sostiene que dichas instituciones gobiernan las interacciones tanto de estados como de actores no-estatales en áreas como el medio ambiente o los DD.HH. Por ejemplo, el mercado mundial de café, es gobernado por una variedad de tratados, acuerdos comerciales, protocolos científicos y de mercado, y los intereses de productores, consumidores y distribuidores. Así pues, los estados organizan estos intereses y consideran las prácticas, reglas y procedimientos para crear un acuerdo de gobierno o régimen que controle la producción del café, monitoreé su distribución y finalmente determine el precio para los consumidores (Young, 1997, citado en, Lamy, 2001: 189).

${ }^{4}$ El autor manifiesta que, a pesar de que el Consejo de Seguridad de la ONU ha expedido resoluciones contra el terrorismo, no ha tenido la capacidad de definirlo. En este sentido, el autor expresa que la definición que le parece más apropiada hasta el momento es la siguiente, de Alex Schmid: «Un método muy angustiante de acciones violentas repetidas, utilizado por un individuo o grupo de actores estatales (semi) clandestino por motivos idiosincráticos, criminales o políticos, por el cual-al contraste del asesinato-los blancos directos de la violencia no son el objetivo principal» (Schmid, 1993, citado en, Keohane, 2002: 30). En cuanto a las consecuencias directas del 11-s en la política domestica de EE.UU. me parece oportuna la definición de Buzan, sobre todo por el tema de libertades y derechos individuales: «[...] el terrorismo, al igual que otras formas de violencia política, no sólo afecta directamente la seguridad individual, también incrementa las amenazas provenientes del propio estado y de los demás, [...] los terroristas pueden forzar al estado a adoptar medidas más molestas» (Buzan, 1993: 41). También la definición del FBI: «Uso de la fuerza por fuera de la ley contra personas o la propiedad para intimidar o coaccionar un gobierno, la población civil, con propósitos políticos o sociales», y la del Departamento de Estado de EE.UU.: «Violencia premeditada, políticamente motivada contra objetivos no-combatientes, por parte de grupos subnacionales o agentes clandestinos, con el propósito de influir en la audiencia», evidencian la dificultad de llegar a una sola definición del fenómeno y sustentan lo expuesto por Keohane anteriormente. 
que se deben revisar varios aspectos, que luego del 11-s adquieren mayor relevancia: I) la obsoleta forma de entender la seguridad, enmarcada en un espacio geográfico, II) la globalización de la violencia informal, que puede ser analizada a través de modelos de interdependencia asimétrica y sus implicaciones de poder, y III) la respuesta de EE.UU. a los ataques del World Trade Center puede explicar, en parte, el papel de las instituciones multilaterales en la política mundial contemporánea (Keohane, 2002: 30).

De acuerdo con lo anterior, el autor insiste en que la globalización de la violencia no es nueva ni tampoco producto del 11-s, pero destaca el incremento en su magnitud como resultado de la reducción de costos en la comunicación y transporte a nivel global, aspectos que con Nye desarrollaron en su trabajo más conocido a finales de la década de 1970 y que continúan demostrando su validez (Keohane y Nye, 1977). Este autor considera que los teóricos actuales de política internacional afrontan los mismos desafíos de una generación atrás: «el espacio geográfico que había sido visto como una barrera natural ahora debe ser visto como un portaaviones también» (Keohane, 2002: 32). Este punto es importante, puesto que el pensamiento realista tradicional en EE.UU. ${ }^{5}$ siempre había impuesto limitaciones a la hora de intervenir en el extranjero, bajo la premisa del interés nacional y de un espacio geográfico establecido; pero ahora EE.UU. corre el riesgo de intervenir en una variedad de conflictos que tal vez tengan poca relación con el «terrorismo» de alcance global.

Un ejemplo puede sustentar lo anterior: Afganistán, en términos realistas, puede ser el lugar menos importante para la política exterior de EE.UU., más aún, luego del colapso de la ex URSS, pero luego del 11-s, en octubre de 2001 se convirtió en un teatro de guerra, y esto se debe a la expansión y eficacia de redes interdependientes e informales que demuestran que las amenazas al territorio de cualquier país pueden provenir de diversas partes del mundo, y es así que la "violencia informal globalizada» hace ver anacrónica la concepción de espacio geográfico tradicional (Keohane, 2002: 33).

Por consiguiente, la transmisión de violencia informal con alcance global ha hecho evidente la vulnerabilidad física de EE.UU., pero más aún, la paradójica debilidad de una «sociedad de la información», en la que antes del 11-s se sabía poco sobre las redes terroristas que operaban dentro de territorio estadounidense, mientras que éstas sí tenían información adecuada sobre sus objetivos, es a lo que Keohane llama «asimetría en la información». ${ }^{6}$ En el mismo orden, el autor acuña el concepto «asimetría en las creencias», puesto que los ataques suicidas de organizaciones como la

\footnotetext{
${ }^{5}$ Clásicos autores realistas de la escuela americana como Walter Lippmann, Hans J. Morgenthau y Kenneth Waltz, fueron fuertes opositores de la intervención militar estadounidense en Vietnam, es lógico pensar que por falta de argumentos claros a favor del interés nacional.

${ }^{6}$ La crítica de Keohane es más radical aún, el autor cita un trabajo de Ole Holsti en el que argumenta que durante varias reuniones del Chicago Council on Foreign Relations, llevadas a cabo en 1994 y 1998, el público se mostraba preocupado por el terrorismo internacional. En la primera reunión el $69 \%$ del público veía al terrorismo como un tema crítico, mientras que sólo el 33\% de la élite lo veía así; en 1998 la relación fue de $84 \%$ y $61 \%$ respectivamente (Holsti, 2000: 21, citado en, Keohane, 2002: 34)
}

WALTZY KEOHANE SOBRE EL 11-SY LA INTERVENCIÓN EN IRAK 
de Bin Laden hacen más complejo prevenir su sorpresivo y destructivo poder (Keohane, 2002: 35). Los anteriores ejemplos de asimetría anulan la mayor cantidad de recursos militares, económicos, políticos, tecnológicos y estratégicos que tiene EE.UU., debido a que grupos terroristas medianos o pequeños pueden ocasionar daños letales, y si durante la década de $1970 \mathrm{EE} . U \mathrm{U}$. fue vulnerable al petróleo, ahora lo es al terrorismo.

Para un institucionalista como Keohane, la respuesta de EE.UU. luego del 11-s es preocupante, puesto que la reacción de Washington se centró en temas como la seguridad doméstica, respuestas militares y la creación de una coalición no muy democrática contra el terrorismo. Peor aún, poca atención se le prestó a la Resolución 1368 del Consejo de Seguridad de la ONU, el día 12 de septiembre de 2001; tampoco el presidente Bush hizo mención a la ONU durante su discurso el día 20 de septiembre, habló sobre la OTAN e hizo una referencia genérica sobre las organizaciones internacionales, y finalmente la cobertura de la prensa hacia la reacción de la ONU fue casi nula; en el New York Times fue prácticamente inexistente (Keohane, 2002: 35).

No obstante, Keohane expresa que el 11-s constituyó una prueba al institucionalismo, ya que antes de los atentados la administración estadounidense había adelantado una política unilateral en varios aspectos, tales como el calentamiento global, el lavado de dinero, tráfico de armas ligeras y evasión de impuestos, temas en los que se dejó de lado a las instituciones internacionales. A pesar de lo anterior, el 28 de septiembre de 2001, EE.UU. se acercó al Consejo de Seguridad de la ONU para adoptar la Resolución 1373, que hacía alusión al Capítulo VII de la Carta de la ONU, alertando a todos los estados para que rechazaran a estados e individuos terroristas, así como también para controlar la financiación del terrorismo. (Keohane, 2002: 36). Esto demuestra que los estados buscan lo que Inis Claude Ilamó «legitimación colectiva», tal vez porque sólo la ONU puede dar base global a una política de estado, que en este caso ha sido nefasta para el orden de la política mundial del siglo XXI. ${ }^{7}$

\subsection{La respuesta de la administración Bush, según Keohane}

Luego del 11-s, Keohane identifica tres fases en la respuesta de la administración Bush: la primera es la de Afganistán, que él considera «normal» porque es la forma en que opera un gran poder; además, Washington contaba con el respaldo de la ONU y se trataba de un caso típico de autodefensa con el que se buscaba destruir bases de terroristas; y aunque no era una contestación suficiente, gran parte del pueblo americano estuvo de acuerdo: «Esa fase es consistente con lo que cualquier administración estadounidense hubiera hecho» (Keohane, 2004).

La segunda fase es una mezcla entre «Realismo» y «Neo-Conservatismo», cuando se convencieron de cambiar y mejorar al mundo atacando a Irak, a sabiendas de que no tenía ningún vínculo con Afganistán, el autor afirma al respecto: «Ni siquiera ellos creían eso, la historia de las WMD fue

${ }^{7}$ (Claude, 1967, citado en, Keohane, 2002: 36). 
algo sobredimensionado, si no inventado» (Keohane, 2004). Incluso, el autor manifiesta, tal y como vimos en apartados anteriores, que algunos realistas se opusieron a la intervención porque para estos las WMD no eran un problema porque muchos países ya las tenían y por lo tanto no era necesario invadir a Irak, y expresa: "I said this before the war, that it was like Thucydides's discussion of the Peloponnesian War: the real cause was the one kept most out of sight» (Keohane, 2004). En este sentido el autor expresa que la verdadera causa de la intervención era demostrar el poder de EE.UU. en Oriente Medio para intimidar a los palestinos o a los iraníes, libios, sirios y demás pueblos de la región, lo cual representa una línea de realismo ofensivo puro de personajes como Rumsfeld y Cheney; otros como Wolfowitz pensaron que podrían instaurar la democracia en Irak y que, además, sería un proceso fácil. Y en este sentido, para Keohane lo más grave está en la forma como se relegó a la ONU, puesto que EE.UU. manifestó que con o sin los votos atacarían a Irak; Rumsfeld lo manifestó: «If we can get the UN on board for our mission, fine, but we're not going to let the coalition determine the mission, $[\ldots]$ and so we're going to disdain the UN if it won't go along» (Keohane, 2004).

Y la tercera fase se hace evidente entre septiembre y octubre de 2003, cuando los problemas en Irak se agudizaban junto con la falta de legitimidad de EE.UU., en ese momento el teórico criticaba que no era posible siquiera establecer un gobierno democrático y ligeramente estable, que no fuera base para terrorismo, que no cayera en una guerra civil y que no fuera opuesto totalmente a EE.UU., y todo lo anterior es lo que efectivamente ha ocurrido. En este sentido, Keohane expresa que las metas que la administración Bush se trazó en Irak son imposibles de cumplir sin la ayuda de la ONU, y es por ello que: «Paul Bremer fue a la oficina de Kofi Annan y le rogó para que hiciera el reporte en el momento de las elecciones»; no obstante, lo más criticable para Keohane fue la impotencia para aceptar los errores: "They'll never say they were wrong; they haven't admitted being wrong to anything. But they have to, in effect, admit that they have to go back to the United Nations» (Keohane, 2004). Y es en esta parte que el autor vuelve a destacar la importancia de las instituciones, porque para él, una vez iniciada la intervención en Irak, la administración Bush se dio cuenta de que necesitaba a la ONU para legitimar su coalición frente al resto de democracias en el mundo. Esta es la lección que deja la intervención en Irak, según Keohane, que las instituciones internacionales son «terriblemente» importantes, incluso en temas de seguridad y high politics, como lo ha sido la intervención americana en Irak desde 2003.

\section{CONCLUSIONES}

Es evidente que en el debate neo-neo se sufre una breve distensión, al analizar las consecuencias del 11-s y la intervención militar de EE.UU. en Irak, a partir de los postulados de Waltz y Keohane. Ambos autores son críticos de las acciones tomadas por la administración Bush. Waltz, por su parte, hace más énfasis en el desequilibrio de poder en el sistema internacional, ocasionado por las decisiones erróneas de Washington luego del 11-s, la proliferación nuclear y las «crisis» en las que se ve inmiscuido EE.UU. En este sentido, se pueden evidenciar los errores en percepción de la 
administración estadounidense, pero también las falacias que se utilizaron para vincular a Al Qaeda y Bin Laden con Hussein, quien para Waltz era un «sobreviviente» del sistema internacional.

El profesor Keohane es más crítico respecto al actuar de la administración Bush. Critica textualmente a miembros de la administración Bush, como Rumsfeld y Rice, argumentando que con sus decisiones unilaterales violaron la normatividad e institucionalidad internacionales. Asimismo, habla sobre la liberalización del miedo y la globalización de la violencia informal, lo cual es producto de la efectividad de las redes de información a disposición de grupos terroristas transnacionales, que pueden ser muy efectivos operando desde failed states, como Afganistán.

Hace casi treinta años Waltz presagió la diseminación de las armas nucleares, y luego del 11-s de 2001 manifestó que son la opción de los estados pequeños para enfrentar al hegemón. Lo anterior es preocupante, pero también la respuesta unilateral de EE.UU. hizo mucho daño a las organizaciones y regímenes internacionales, que autores como Keohane ayudaron a fortalecer epistemológicamente desde la academia.

Tal vez el 11-s y la intervención militar en Irak sean los últimos acontecimientos de la reconfiguración política mundial vistos por los ojos de estos autores que, de alguna manera, son un referente para el estudio de la teoría y política mundiales. Y que por lo visto, no están tan alejados como en el fuerte debate que los enfrentó en la década de 1980.

\section{BIBLIOGRAFÍA}

Barbé, E. (2007) Relaciones internacionales, Madrid: Tecnos.

Buzan, B. (1993) «From International System to International Society: Structural Realism and Regime Theory Meet the English School», en International Organization, 47 (3) 327-352.

Claude, I. (1967) Tha Changing United Nations, New York: Random House.

Griffiths, M., Roach, S. y Solomon, S. (1999) Fifty Key Thinkers in International Relations, New York: Routledge.

Haas, P. M., Keohane, R. O. y Levy, M. A. (1993) Institutions for the earth : sources of effective international environmental protection, Cambridge, Mass.: MIT Press.

Holsti, O. (2000) Public Opinion and Foreign Policy. En Lieber, R. (Ed.) Eagle Rules? New York: Longman.

Kennedy-Pipe, C. y Renger, N. (2006) «Apocalype Now? Continuities or Disjunctions in World Politics after 9/ 11.» en International Affairs, 82 (3).

Keohane, R. O. (1982) «The Demand for International Regimes», en International Organization, 36 (2) 325-355.

Keohane, R. O. (1986) Neorealism and it's Critics, Columbia University Press.

Keohane, R. O. (1988) «International Institutions: Two Approaches», en International Studies Quarterly, 32 (4) 379-396.

Keohane, R. O. (1998) «International institutions: Can interdependence work?» en Foreign Policy, 110 (Spring) 82. 
Keohane, R. O. (2002) «The Globalization of Informal Violence, Theories of World Politics, and the «Liberalism of Fear»m, en Dialog-IO, 29-43.

Keohane, R. O. (2004) Theory and International Instituions. Conversations with History; Institute of International Studies, UC Berkeley. Disponible: http://globetrotter.berkeley.edu/people4/Keohane/keohane-con5.html, Acceso: 2 de septiembre de 2008

Keohane, R. O. y Nye, J. (1977) Power and Interdependence: World Politics in Transition, Boston: Little, Brown.

Krasner, S. D. (1983) International regimes, Ithaca: Cornell University Press.

Lamy, S. (2001) Neo Realism and Neo Liberalism. En Baylis, J. y Smith, S. (Eds.) The globalization of world politics : an introduction to international relations. Oxford; New York: Oxford University Press.

Raas, W. A. y Long, A. (2007) «Osirak Redux? Assessing Israeli Capabilities to Destroy Iranian Nuclear Facilities», en International Security, 31 (4) 7-33.

Schmid, A. (1993) The Response Problem as a Definition Problem. En Schmid, A. y Crelinsten, R. (Eds.) Western Responses to Terrorism. London: Frank Cass.

Sodupe, K. (2003) La teoría de las relaciones internacionales a comienzos del siglo XXI, Zarautz: Universidad del País Vasco, Servicio Editorial.

Viotti, P. y Kauppi, M. (1987) International Relations Theory, New York: Mc Millan.

Waltz, K., Sagan, S. y Sigal, L. (1995) The Spread of Nuclear Weapons: A Debate, W. W. Norton \& Company.

Waltz, K. N. (1979) Theory of International Politics, London: Reading, Mass.: Addison-Wesley.

Waltz, K. N. (1988) «The Origins of War in Neorealist Theory Like», en Journal of Interdisciplinary History, 18 (4) 615-628.

Waltz, K. N. (2000) «Structural Realism after the Cold War», en International Security, 25 (1) 5-41.

Waltz, K. N. (2002) The Continuity of International Politics. En K., B. y Dunne (Eds.) Worlds in Collision: Terror and the Future of Global Order. Houndmills, Basingstoke: Palgrave, Macmillan.

Waltz, K. N. (2003) Theory and International Politics. Conversations with History: Institute of International Studies, UC Berkeley. Disponible: http://globetrotter.berkeley.edu/people3/Waltz/waltz-con6.html, Acceso: 26 de agosto de 2008

Weaver, O. (1996) The Rise and Fall of the Inter-Paradigm Debate. En Smith, S., K., B. y M., Z. (Eds.) International Theory: Positivism and Beyond. Cambridge: Cambridge University Press.

Young, O. R. (1997) Global governance : drawing insights from the environmental experience, Cambridge, Mass.: MIT Press. 